\title{
A Multiparameter Biosignal Sensor for Mobile Health Service
}

\author{
Sung Jae Kim, Sangjoon Lee, Jea Hoon Jeong, \\ Sung Yun Park and Sung Min Kim* \\ Department of Medical Biotechnology, Dongguk University-Seoul, 100715 Seoul, South Korea
}

(Received December 24, 2013; accepted March 6, 2014)

Key words: $\mathrm{m}$-Health sensor, biosignal sensor, m-Health, e-Health, tele-health

In this study, a multiparameter biosignal sensor was proposed for the use of smartphones in a mobile health ( $\mathrm{m}$-Health) service application. The proposed sensor for the experiments consisted of one set of two small devices; the size of each sensor was $25 \times$ $20 \times 4 \mathrm{~mm}^{3}$. This sensor was made of either copper or titanium to achieve better body affinity and electric conductivity. The manufactured sensor was able to measure major bioparameters including the electrocardiogram (ECG) signal of a channel, temperature, photoplethysmography (PPG), and body fat. Furthermore, an independent analog hardware was manufactured to measure each bioparameter. Our results showed that bioparameters were easily measured and they will be effectively applied to smartphones.

\section{Introduction}

The medical paradigm has been changing, in which patients are considered as active consumers, not passive, since there are many changes in the socioeconomy as well as increasing interests in health. Along with such changes in life patterns, ubiquitous services (u-services) are expanding via ubiquitous technologies in society, thereby requiring $\mathrm{m}$-health services for better medical service satisfaction as well as quality of life of people, and designing various electronic health (e-Health) terminals. ${ }^{(1,2)}$ Ubiquitous sensor network (USN)-based terminals allow various types of terminal connection. In particular, it is important for biosignal measurement and device design to monitor biosignals such as electrocardiogram signals, which need to be monitored for $24 \mathrm{~h}$ and to observe patients anywhere and anytime. Previously, m-Health services were provided via specific terminals with medical servers and data communication utilizing Wi-Fi, whereas biosignal measurement devices were provided using either a personal area network (PAN) or a body area network (BAN) so that terminal and biosignal data were able to communicate with each other. ${ }^{(3,4)}$ However, such services require expensive specific terminals and biosignal measurement devices; furthermore, service fees, limitation in migration, and complexity of use were considered factors detrimental to the

${ }^{*}$ Corresponding author: e-mail: smkim@dongguk.edu 
vitalization of m-Health services. Recently, smartphones such as iPhones and Android phones, have been coming into widespread use, allowing network connection anytime and anywhere, thereby providing a much better platform for health management via USN; it can be considered that the platform of the m-Health service via USN is already set. To realize the m-Health service on smartphones, methods are needed to measure the biosignal, and the following conditions should be satisfied to expect the vitalization of m-Health.

1) The measurement of biosignals should not be restrictive for the user's convenience.

2) The measurement electrode should not be attached to the body for a long time.

3) Multiple biosignals should be measured with a single measurement.

4) The measurement hardware should be ultrasmall so that it can be attached to smartphones as an accessory.

5) While measuring or right after the measurement, data should be analyzed automatically.

6) Data storage and transmission should be carried out automatically.

7) Operation should be simple for the elderly.

8) Security functions should be added to protect health information. ${ }^{(5)}$

Items 1) through 4) are related to measurement sensors and hardware, whereas items 5) to 8 ) are concerned with measurement terminals that can be easily solved if smartphones are utilized. To resolve items 1) to 4), the multiparameter biosignal measurement sensor was proposed in this study. The manufactured sensor consists of one set of two small devices; the size of each sensor was $25 \times 20 \times 4 \mathrm{~mm}^{3}$. This sensor was made of either copper or titanium to achieve better body affinity and electric conductivity. A manufactured sensor was able to measure four major bioparameters including the electrocardiogram (ECG) signal of a channel, temperature, PPG, and body fat. An independent analog ultrasmall hardware was manufactured to measure each bioparameter and the signal was tested. Therefore, the smartphone m-Health monitoring system can be organized via the system proposed in this study, and eventually, it will help vitalize the m-Health dissemination system.

\section{Materials and Methods}

\subsection{Multiparameter biosignal measurement sensor}

The multiparameter biosignal measurement sensor proposed in this study is shown in Fig. 1; one set consists of the left and right sensors. As shown in Fig. 1(a), the left and right sensors are placed horizontally, and both fingers touch those sensors so that the biosignals can be measured. For the part contacting both forefingers, it was processed in a round shape to increase the surface area, thereby increasing the measurement accuracy.

For the sensor material, it was made of either copper or titanium to increase the bioaffinity as well as electric conductivity. The right-side sensor is a rectangular hole provided to place the PPG sensor and consists of a round hole in the rear side to place the temperature sensor, which is arranged to fix into the PCB. Figure 1(b) shows the measurement method for multiparameter biosignals; once the sensor is placed on the PCB, select the ECG and body fat measurement mode via the analog switch 
of the control part. The sensor proposed in this study was designed to measure both temperature and PPG simultaneously when either ECG or body fat is measured.

Figure 2 shows the hardware block diagram designed for multiparameter biosignal measurement via the sensor proposed in this study; it consists of a sensor, an amplification part, a signal-filtering part, an analog-to-digital (A/D) converter, an A/D channel multiplexer, a microprocessor, and a communication interface part. Considering the power and power consumption, the power of an analog circuit as well as digital circuit was designed for $5 \mathrm{~V}$ operation; a $3.3 \mathrm{~V} 300 \mathrm{~mA} / \mathrm{H}$ lithium polymer battery was built in so that it is able to increase the power to $5 \mathrm{~V}$. For the communication with smartphones, near-field communication (NFC) was designed; furthermore, RS-232 and Zigbee communication functions were also added to debug the measured signals.

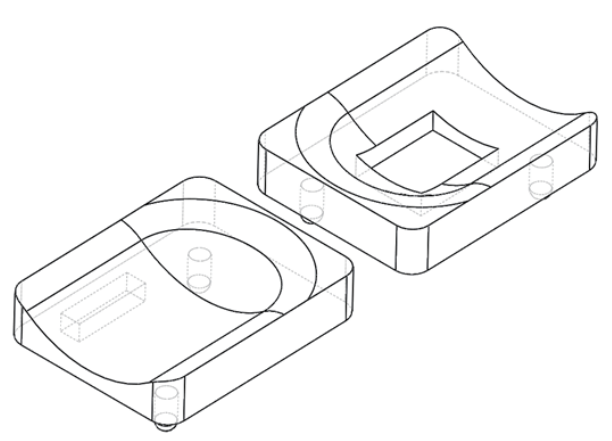

(a)

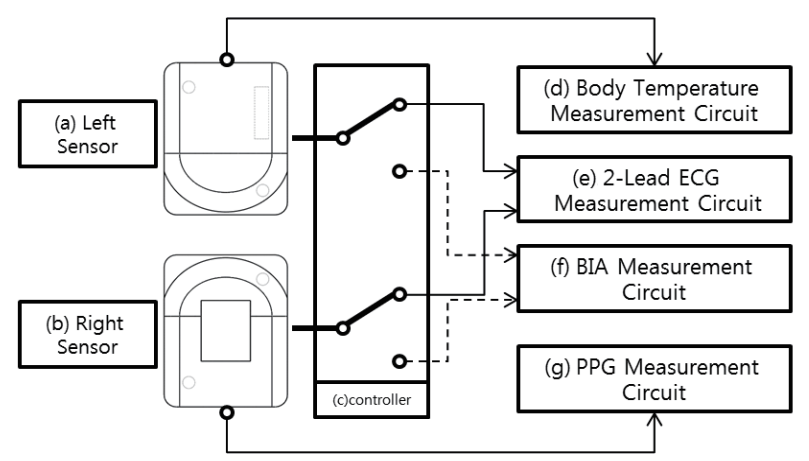

(b)

Fig. 1. Sensor design and multiparameter biosignal measurement method: (a) multiparameter biosignal measurement sensor and (b) multiparameter biosignal measurement method.

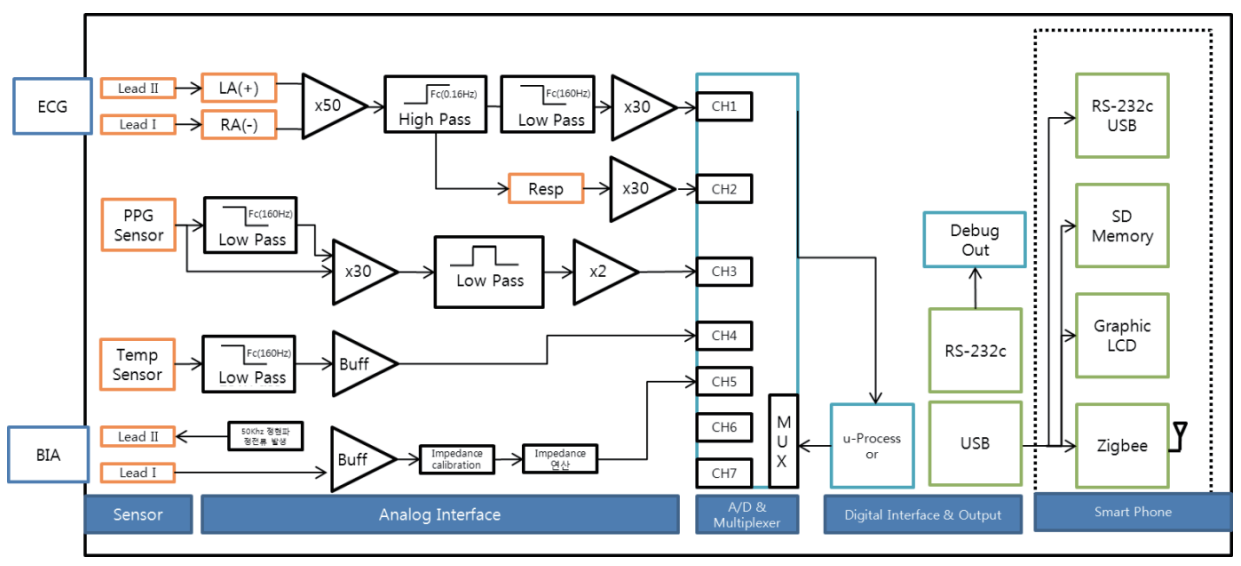

Fig. 2. (Color online) Manufactured multiparameter biosignal measurement hardware block diagram. 


\subsection{Two-electrode ECG measurement}

The ECG is an electrical conduction phenomenon occurring in the heart and generated by the action potential of heart cells. To measure such electrical activities in the heart, a 3-lead measurement method was firstly proposed by Einthoven in 1908, and now, 12 leads are used to measure the multichannel ECG for better observation. The minimum number of electrodes is 3 to measure the ECG, and currently, the use of fewer electrodes is being investigated in $\mathrm{m}$-Health and e-Health devices for the general public, not for cardiologists. In this study, a high-pass filter $\left(F_{\mathrm{c}}=0.05 \mathrm{~Hz}\right)$ and a low-pass filter were added and applied on the top of the hardware for the 2-lead electrodes, which was proposed by Dobrev et al. ${ }^{(6)}$

\subsection{PPG measurement}

In general, PPG is frequently utilized to measure the velocity of the blood stream as well as changes in the volume of blood vessels when the blood reaches the limbs from the heart; the wavy pattern is similar to that of arterial blood pressure. It is applicable to measure blood flow from the fingertips and toe tips of limbs, radial artery, and ears..$^{(7)}$ Although there are several measurement methods available for plethysmography (e.g., ultrasound, photodiode, and laser), the photodiode is widely used since it is easy to fabricate and inexpensive. The plethysmogram measurement using the photodiode called PPG and a wavy pattern can be obtained by transmitting a light source with the range of 660-1000 $\mathrm{nm}$ on a certain location. Application fields include diagnosis of sclerosis of the arteries by calculating the elasticity of blood vessels since it is possible to monitor the velocity of the blood stream through the changes in the volume of blood vessels. The sensor type for the measurement of PPG, which is a clip-type sensor, is commonly used, while there are various measurement types of sensor such as a glass type, inner-ear measurement type, and an earlobe measurement type. The sensor proposed and utilized in this study is the reflection-type PPG sensor shown in Fig. 3(a), which can be located on the right side of the rectangular hole (DCM02, AMPKorea, Inc.).

\subsection{Measurement of body fat}

The measurement of body fat is one of the methods of measuring the degree of obesity, and the amount of fat is calculated on the basis of one's height and weight. The

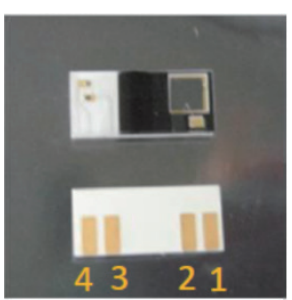

(a)

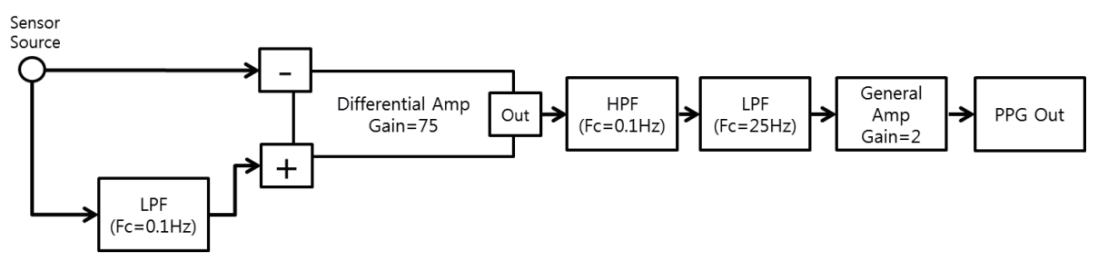

(b)

Fig. 3. (Color online) PPG sensor and hardware block diagram: (a) PPG sensor for reflection type and (b) analog block diagram for acquiring PPG. 
device for body fat measurement mostly utilizes the high electric resistance of fat. The electric resistances of various components of the body, such as protein and fat, are all different. Water, comprising $70 \%$ of the body, allows electricity to flow very well. Fat, however, does not allow electricity to flow since it is difficult for ions and electrons to penetrate fats. The device for body fat measurement allows very weak electricity to flow into the body and measures the resistance so that body fat can be calculated. The higher the resistance, the more the body fat. This method is called the bio-electroresistance method (bioimpedance method). A precise device for body fat measurement measures the fat of the upper body, lower body, arms, and legs separately. There are several other methods of measuring body fat, such as skinfold measurement (caliper method), which measures the thickness of fat by nipping the abdominal fat using a pair of forceps, and the method that analyzes the spectrum of reflected electronic wave after shooting the ultrasound wave or infrared rays on the fat. ${ }^{(8)}$ In this study, a constant electric current sine wave with a $50 \mathrm{kHz}$ range was generated and transmitted from the right sensor to the left sensor, and then a wavy pattern was measured on the left. By this method, the bioimpedance of the body was measured owing to the electric current path from a right finger to a left finger. Figure 4 shows the block diagram of body impedance analysis (BIA) while Fig. 4(A) shows the source of constant electric current for the measurement of the impedance of body fat. Figures 4(B) and 4(C) show the resistance for the calculation of impedance of the circuit, and this resistance was selected within the range of body impedance; in this circuit, Block B (100 $\Omega$ ) and Block C (1000 $\Omega$ ) were used. Figure $4(\mathrm{E})$ shows the analog multiplexer controller and the switching is performed in the order of (B), (C), and (D). In Fig. 4, the output signal of Block E will measure the voltage as the amplitude of a sine wave of $50 \mathrm{kHz}$ becomes smaller and is flattening the output signal corresponding to the impedance. As the voltage can be calculated on the basis of the impedances of Blocks B and C, the bioimpedance can be easily calculated using the linear interpolation calculation.

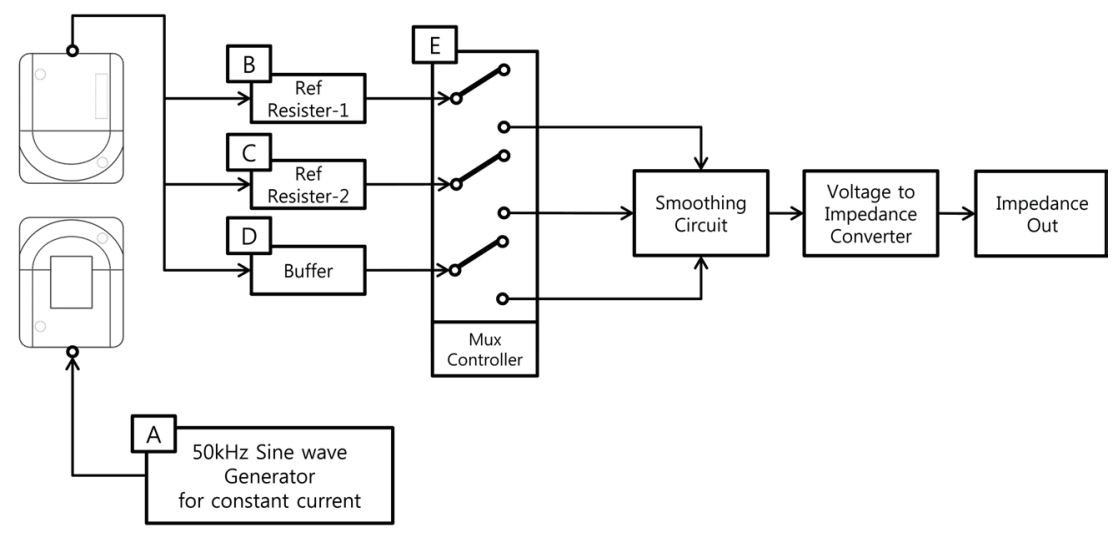

Fig. 4. Body impedance analysis analog bock diagram. 


\subsection{Temperature measurement}

Temperature is one of the most important bioparameters that represent vitality, and there are several different thermometers for medical purposes, such as the infrared ray thermometer, mercury thermometer, and thermometers utilizing NTC or PTC. To measure the temperature of a deep part of the body, a rectal thermometer, which measures temperature directly, and a thermometer that measures temperature on the skin and corrects it by utilizing the characteristic curve of specific parts of the body are widely used. In this study, a double-heat-sink diode-type temperature sensor (Thermometric, TH410J40G model) was used, and as shown in Fig. 5, it represents the linear relationship between temperature and changes in resistance in a certain range $\left(25-40{ }^{\circ} \mathrm{C}\right)$. The thermosensor was mounted on the groove of a proposed sensor in this study and designed to transmit heat without heat loss by utilizing heat sink grease, which exhibits excellent heat transfer characteristics.

\section{Experiment}

Figure 6(a) shows the actual manufactured sensor and Fig. 6(b) reveals the hardware mount with the manufactured sensor. The hardware size for the experiment was $55 \times$

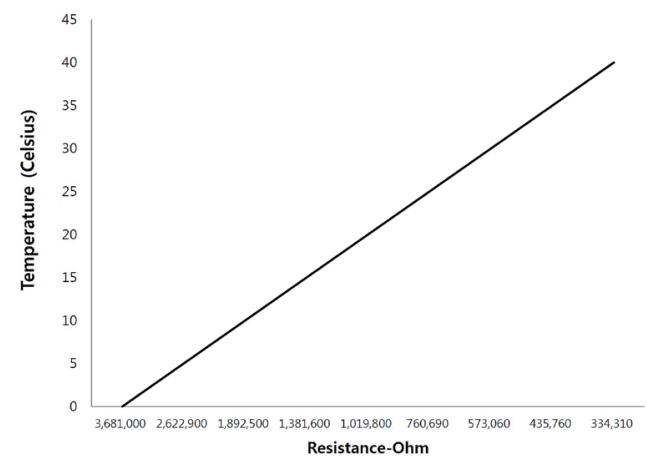

Fig. 5. PTC temperature-resistance characteristic curve (TH410J40G-Themometric, Inc.).

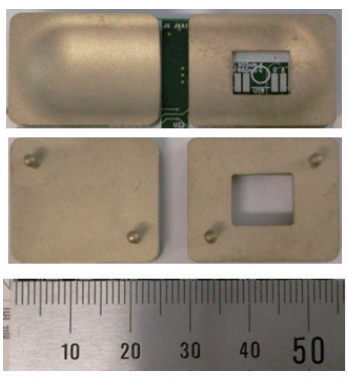

(a)

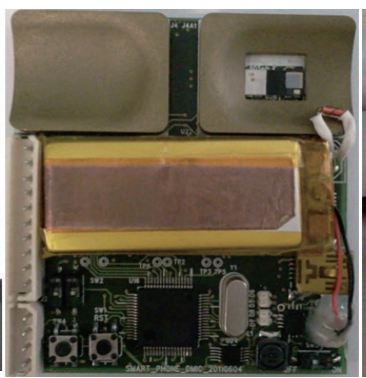

(b)

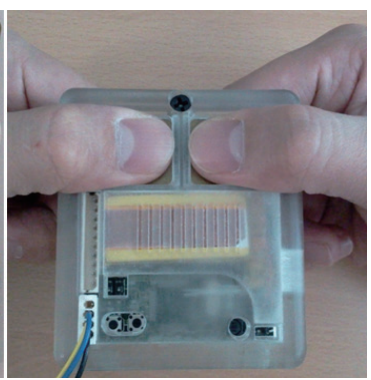

(c)

Fig. 6. (Color online) Sensors and actual designed hardware for testing: (a) an actual designed sensor, (b) testing hardware, and (c) snapshot for experiment of multi-biosignal measurement. 
$50 \mathrm{~mm}^{2}$; to be applicable as a type of smartphone accessory, it should have an ultrasmall size. As shown in Fig. 6(c), the program was designed for subjects to measure either (electrocardiogram mode: 1 channel electrocardiogram + temperature + PPG) or (body fat mode: body fat + temperature + PPG) by putting their forefingers on the sensor.

For analyzing the ECG and PPG signal accuracies between the proposed method and the conventional measurement method (BIOPAC System, Inc., MP150 ECG and PPG kit), we acquired the ECG and PPG signals from 10 subjects for 3 min at the same time and then extracted heartbeats from the obtained ECG and PPG signals. We then investigated the correlation factor between the two extracted heartbeats. The average correlation factor is 0.9911 , as shown in Fig. 7. Also, we have tested the body fat impedance accuracy using a carbon film resistor in the range of $100 \Omega-1 \mathrm{k} \Omega(100,200$, $330,430,560,680,750,820,910$, and $1 \mathrm{~K})$. The experiment results showed that a high accuracy of $96.8 \%$ was obtained.

Figure 8 shows the PC screen when in the electrocardiogram mode. In the experiments, 10 individuals were subjected to measurements in both ECG mode and body fat mode for $3 \mathrm{~min}$ each; in each mode, biological data were measured effectively.

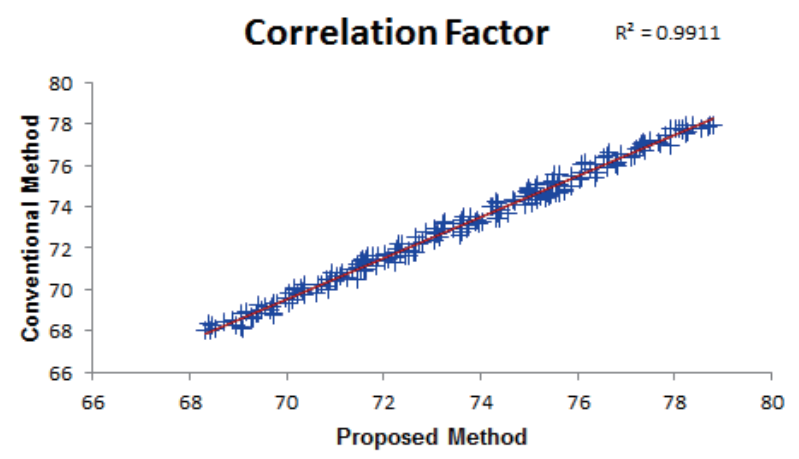

Fig. 7. (Color online) Heartbeat correlation factor between conventional and proposed measurement methods (ECG and PPG).

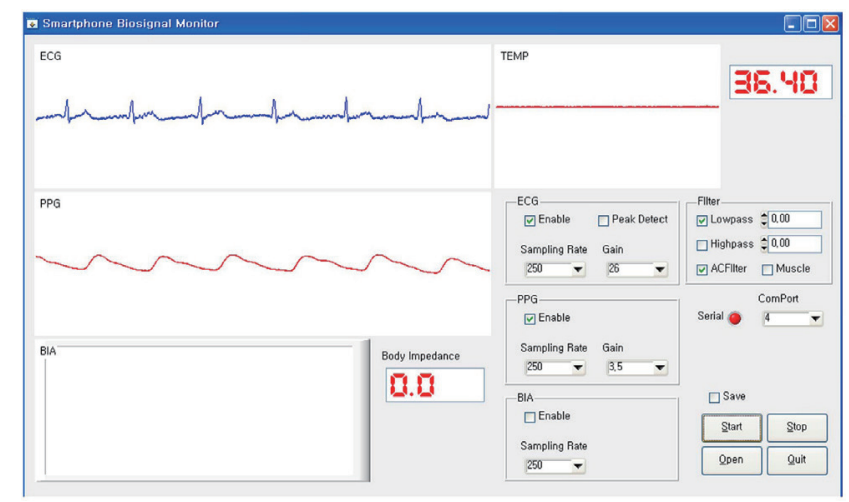

Fig. 8. (Color online) Biosignal measurement monitoring program snapshot. 


\section{Conclusion and Discussion}

In this study, a sensor and related hardware were manufactured to acquire multiparameter biosignals that allow the applications of smartphone m-Health. Results showed that the manufactured sensor was able to measure biosignals, including ECG, PPG, body fat, and temperature simultaneously. If this sensor and hardware are applied on smartphones and then applications are developed, it enables (1) effective individual health management (heart disease diagnosis, partial management of sclerosis of the arteries via electrocardiogram and PPG, management of body fat and obesity, and temperature management), (2) transmission of vital signs to hospitals and doctors under emergency situations, and (3) live monitoring of emergency issues of subjects and immediate early countermeasures for such issues. Particularly, it will be helpful for the elderly who live alone to prevent solitary deaths. Furthermore, such devices will be applicable for general households, ambulances, and nursing homes, as well as for monitoring individual patients in mid to large-sized hospitals; in the future, it will also be utilized as an effective device for m-Health monitoring.

\section{Acknowledgements}

This study was supported by a grant from the Korea Healthcare Technology R\&D Project, Ministry of Health \& Welfare, Republic of Korea (A102058).

\section{References}

1 M. Cattan, M. White, J. Bond and A. Learmouth: Ageing \& Society 25 (2005) 41.

2 B. G. Celler, W. Earnshaw, E. D. Ilsar, L. Betbeder-Matibet, M. F. Harris, R. Clark, T. Hesketh and N. H. Lovell: Int. J. Bio-Med. Comput. 40 (1995) 147.

3 S. J. Lee, J. K. Kim and M. H. Lee: Telemed. J. E. Health 17 (2011) 123.

4 J. A. Gutierrez, M. Naeve, E. Callaway, M. Bourgeois, V. Mitter and B. Heile: IEEE Network 15 (2001) 12.

5 S. J. Lee, J. K. Kim and M. H. Lee: IEEE Trans. Biomed. Eng. 58 (2011) 2448.

6 D. Dobrev, T. Neycheva and N. Mudrov: Medical \& Biological Engineering \& Computing 42 (2005) 725.

7 J. Allen: Physiol. Meas. 28 (2007) R1.

8 R. F. Kushner: J. Am. Coll. Nutr. 11 (1992) 1999. 